\title{
Dark matter voids in the SDSS galaxy survey
}

\author{
Florent Leclercq, 1, 2, 3, a) Jens Jasche, 1,2 P. M. Sutter, 1, 2, 4, 5, 6 Nico Hamaus, ${ }^{1,2}$ and Benjamin Wandelt ${ }^{1,2,7,8}$ \\ ${ }^{1)}$ Institut d'Astrophysique de Paris (IAP), UMR 7095, CNRS - UPMC Université Paris 6, Sorbonne Universités, \\ 98bis boulevard Arago, F-75014 Paris, France \\ ${ }^{2)}$ Institut Lagrange de Paris (ILP), Sorbonne Universités, \\ 98bis boulevard Arago, F-75014 Paris, France \\ 3) École polytechnique Paris Tech, \\ Route de Saclay, F-91128 Palaiseau, France \\ ${ }^{4)}$ Center for Cosmology and Astro-Particle Physics (CCAPP), The Ohio State University, \\ 191 West Woodruff Avenue, Columbus, OH 43210, USA \\ ${ }^{5)}$ National Institute for Nuclear Physics (INFN), \\ via Valerio 2, I-34127 Trieste, Italy \\ ${ }^{6)}$ Osservatorio Astronomico di Trieste (INAF), \\ via Tiepolo 11, I-34143 Trieste, Italy \\ 7) Department of Physics, University of Illinois at Urbana-Champaign, \\ 1110 West Green Street, Urbana, IL 61801, USA \\ ${ }^{8)}$ Department of Astronomy, University of Illinois at Urbana-Champaign, \\ 1002 West Green Street, Urbana, IL 61801, USA
}

(Dated: 15 June 2018)

What do we know about voids in the dark matter distribution given the Sloan Digital Sky Survey (SDSS) and assuming the $\Lambda$ CDM model? Recent application of the Bayesian inference algorithm BORG to the SDSS Data Release 7 main galaxy sample has generated detailed Eulerian and Lagrangian representations of the largescale structure as well as the possibility to accurately quantify corresponding uncertainties. Building upon these results, we present constrained catalogs of voids in the Sloan volume, aiming at a physical representation of dark matter underdensities and at the alleviation of the problems due to sparsity and biasing on galaxy void catalogs. To do so, we generate data-constrained reconstructions of the presently observed large-scale structure using a fully non-linear gravitational model. We then find and analyze void candidates using the VIDE toolkit. Our methodology therefore predicts the properties of voids based on fusing prior information from simulations and data constraints. For usual void statistics (number function, ellipticity distribution and radial density profile), all the results obtained are in agreement with dark matter simulations. Our dark matter void candidates probe a deeper void hierarchy than voids directly based on the observed galaxies alone. The use of our catalogs therefore opens the way to high-precision void cosmology at the level of the dark matter field. We will make the void catalogs used in this work available at http://www.cosmicvoids.net.

\section{INTRODUCTION}

Observations of the cosmic large-scale structure (LSS) have revealed that galaxies tend to lie in thin wall-like structures surrounding large underdense regions known as voids, which constitute most of the volume of the Universe. Although the discovery of cosmic voids dates back to some of the first galaxy redshift surveys (Gregory \& Thompson, 1978; Kirshner et al., 1981; de Lapparent, Geller \& Huchra, 1986) and their significance was assessed in some early studies (Martel \& Wasserman, 1990; van de Weygaert \& van Kampen, 1993; Goldberg \& Vogeley, 2004), the systematic analysis of void properties has only been considered seriously as a source of cosmological information in the last decade (e.g. Sheth \& van de Weygaert, 2004; Colberg et al., 2005; Viel, Colberg \& Kim, 2008; Betancort-Rijo et al., 2009; Lavaux \& Wandelt, 2010; Biswas, Alizadeh \& Wandelt, 2010; van de Weygaert \& Platen, 2011; Lavaux \& Wandelt, 2012, and references therein). Like overdense tracers of the density

\footnotetext{
a)Electronic mail: florent.leclercq@polytechnique.org
}

field such as clusters, voids can be studied by statistical methods in order to learn about their distribution and properties compared to theoretical predictions.

Generally, direct sensitivity of void statistics to cosmology is only guaranteed for the underdense regions of the overall matter density field, which includes a large fraction of dark matter (DM). These are the physical voids in the LSS, for which theoretical modeling is established. However, absent direct measurements of dark matter underdensities, current void catalogs are defined using the locations of galaxies in large redshift surveys (Pan et al., 2012; Sutter et al., 2012b, 2014c; Nadathur \& Hotchkiss, 2014). Since galaxies trace the underlying mass distribution only sparsely, void catalogs are subject to uncertainty and noise. Additionally, numerical simulations show that there exists a population of particles in cosmic voids. This is an indication of physical biasing in galaxy formation: there is primordial dark and baryonic matter in voids, but due to the low density, little galaxy formation takes place there. Additionally, due to complex baryonic physics effects during their formation and evolution, galaxies are biased tracers of the underlying density field, which gives rise to qualitatively different void properties. 
The sensitivity of void properties to the sampling density and biasing of the tracers has only been recently analyzed in depth on simulations, by using synthetic models to mimic realistic surveys. Little \& Weinberg (1994); Benson et al. (2003); Tinker \& Conroy (2009); Sutter et al. (2014a) found that the statistical properties of voids in galaxy surveys are not the same as those in dark matter distributions. At lower tracer density, small voids disappear and the remaining voids are larger and more spherical. Their density profiles get slightly steeper, with a considerable increase of their compensation scale, which potentially may serve as a static ruler to probe the expansion history of the Universe (Hamaus et al., 2014b). Hamaus, Sutter \& Wandelt (2014) recently proposed a universal formula for the density profiles of voids, describing in particular dark matter voids in simulations (see also Colberg et al., 2005; Paz et al., 2013; Ricciardelli, Quilis \& Varela, 2014; Nadathur et al., 2014). The connection between galaxy voids and dark matter voids on a one-by-one basis is difficult due to the complex internal hierarchical structure of voids (Dubinski et al., 1993; van de Weygaert \& van Kampen, 1993; Sahni, Sathyaprakah \& Shandarin, 1994; Sheth \& van de Weygaert, 2004; Aragon-Calvo \& Szalay, 2013; Sutter et al., $2014 c, b)$. However, the nature of this relationship determines the link between a survey, with its particular tracer density, and the portion of the cosmic web that it represents. Understanding this connection is of particular importance in light of recent results which probe the LSS via its effect on photons geodesics. These results include Melchior et al. (2014); Clampitt \& Jain (2014), which probe the dark matter distribution via weak gravitational lensing; Ilić, Langer \& Douspis (2013); Planck Collaboration (2014) for the detection of the integrated Sachs-Wolfe effect in the cosmic microwave background, sensitive to the properties of dark energy. As a response to this demand, Sutter et al. (2014b) found that voids in galaxy surveys always correspond to underdensities in the dark matter, but that their centers may be offset and their size can differ, in particular in sparsely sampled surveys where void edges suffer fragmentation.

While previous authors offer broad prescriptions to assess the effects of sparsity and biasing of the tracers on voids, the connection between galaxy voids of a particular survey and dark matter underdensities remains complex. In particular, disentangling these effects from cosmological signals in presence of the uncertainty inherent to any cosmological observation (selection effects, survey mask, noise, cosmic variance) remains an open question. In this work, we propose a method designed to circumvent the issues due to the conjugate and intricate effects of sparsity and biasing on galaxy void catalogs. In doing so, we will show that voids in the dark matter distribution can be constrained by the $a b$ initio analysis of surveys of tracers, such as galaxies. We will demonstrate the feasibility of our method and obtain catalogs of dark matter voids candidates in the Sloan Digital Sky Survey Data Release 7.
Our method is based on the identification of voids in the dark matter distribution inferred from large-scale structure surveys. The constitution of such maps from galaxy positions, also known as "reconstruction", is a field in which Bayesian methods have led to enormous progress over the last few years. Initial approaches typically relied on approximations such as a multivariate Gaussian or log-normal distribution for density fields, with a prescription for the power spectrum to account for the correct two-point statistics (Lahav et al., 1994; Zaroubi, 2002; Erdoğdu et al., 2004; Kitaura \& Enßlin, 2008; Kitaura et al., 2009; Kitaura, Jasche \& Metcalf, 2010; Jasche \& Kitaura, 2010; Jasche et al., 2010a,b). However, due to their potentially complex shapes, proper identification of structures such as voids requires reconstructions correct not only at the level of the power spectrum, but also higher-order correlators. Inferences of this kind from observational data have only been made possible very recently by the introduction of physical models of structure formation in the likelihood. This naturally moves the problem to the inference of the initial conditions from which the large-scale structure originates (Jasche \& Wandelt, 2013a; Kitaura, 2013; Wang et al., 2013).

This work exploits the recent application of the BORG (Bayesian Origin Reconstruction from Galaxies, Jasche \& Wandelt, 2013a, BORG method hereafter) algorithm to the Sloan Digital Sky Survey (SDSS) galaxies (Jasche, Leclercq \& Wandelt, 2015, BORG SDSs hereafter), and on the subsequent generation of constrained non-linear realizations of the present large-scale distribution of dark matter. BORG is a full-scale Bayesian framework, permitting the four-dimensional physical inference of density fields in the linear and mildly non-linear regime, evolving gravitationally from the initial conditions to the presently observed large-scale structure. By exploring a highly non-linear and non-Gaussian LSS posterior distribution via efficient Markov Chain Monte Carlo methods, it also provides naturally and fully self-consistently accurate uncertainty quantification for all derived quantities. A straightforward use of reconstructed initial conditions is to resimulate the considered volume (Lavaux, 2010; Kitaura, 2013; Heß, Kitaura \& Gottlöber, 2013). In the same spirit, building upon the inference of the initial conditions by BORG, one can generate a set of dataconstrained realizations of the present large-scale structure via full $N$-body dynamics. As we will show, we make use of initial conditions reconstructed by BORG without any further post-processing, which demonstrates the high quality of inference results.

Due to the limited number of phase-space foldings, the influence of non-linearity in cosmic voids is expected to be milder as compared to galaxies and dark matter halos (Neyrinck, 2012; Neyrinck \& Yang, 2013; Leclercq et al., 2013, see also Abel, Hahn \& Kaehler, 2012; Falck, Neyrinck \& Szalay, 2012; Shandarin, Habib \& Heitmann, 2012). For this reason, voids are more closely related to the initial conditions of the Universe, which makes 
them the ideal laboratories for physical application of Bayesian inference with BORG. In this work, we apply the void finder algorithm VIDE (Sutter et al., 2015b, VIDE hereafter), based on zOBOV (Neyrinck, 2008), to dataconstrained, non-linear reconstructions of the LSS. Each of them is a full physical realization of densely-sampled particles tracing the dark matter density field. In this fashion, we construct catalogs of dark matter voids in the SDSS volume robust to sparsity and biasing of galaxies. As we will show, this procedure drastically reduces statistical uncertainty in void catalogs. Additionally, the use of data-constrained reconstructions allows us to extrapolate the void identification in existing data (e.g. at very small or at the largest scales, at high redshift or near the survey boundary).

As described in BORG method; BORG SDSS, the BORG inference framework possesses a high degree of control on observational systematic and statistical uncertainties such as noise, survey geometry and selection effects. Uncertainty quantification is provided via efficient sampling of the corresponding LSS posterior distribution. The resultant set of initial and final density field realizations yields a numerical representation of the full posterior distribution, capturing all data constraints and observational uncertainties. Building upon these results, in this work, we will extend our Bayesian reasoning to void catalogs. Specifically, we apply full non-linear $N$-body dynamics to a set of data-constrained initial conditions to arrive at a set of non-linear dark matter density fields at the present epoch. As a result, we obtain a probabilistic description of non-linear density fields constrained by SDSS observations. Applying the VIDE void finder to this set of reconstructions yields $N$ data-constrained realizations of the catalog, representing the posterior probability distribution for dark matter voids given observations. In this fashion, we have fully Bayesian access to uncertainty quantification via the variation between different realizations. In particular, we are now able to devise improved estimators for any void statistics by the use of Blackwell-Rao estimators. To assess the robustness of this technique for cosmological application, we focus on three key void observables: number functions, ellipticity distributions and radial density profiles. These are especially sensitive probes of non-standard cosmologies (Bos et al., 2012) and are well understood in both data and simulations (e.g Sutter et al., 2014c).

As a general matter, we stress that these dataconstrained realizations of dark matter void catalogs were obtained assuming a $\Lambda \mathrm{CDM}$ prior. Using our products for model testing therefore requires care: in the absence of data constraints, one will simply be dealing with realizations of the $\Lambda \mathrm{CDM}$ prior. Consequently, any departure from unconstrained $\Lambda$ CDM predictions are driven by the data. Conversely, for model tests where the data are not strongly informative, agreement with $\Lambda \mathrm{CDM}$ is the default answer.

This paper is organized as follows. In section II, we describe our methodology: Bayesian inference with the
BORG algorithm, non-linear filtering of the results, void identification technique and Blackwell-Rao estimators for void statistics. In section III, we examine the properties of the dark matter voids in our catalogs. Finally, in section IV we summarize our results, discuss perspectives for existing and upcoming galaxy surveys and offer concluding comments.

\section{METHODOLOGY}

In this section, we describe our methodology step by step:

1. inference of the initial conditions with BORG (section II A),

2. generation of data-constrained realizations of the SDSS volume (section II B),

3. void finding and processing (section II C),

4. combination of different void catalogs with Blackwell-Rao estimators (section IID).

In section IIE, we describe the void catalogs used as references for comparison with our results. These are galaxy void catalogs directly based on SDSS galaxies without use of our methodology, and catalogs of voids in dark matter simulations.

A schematic representation of our procedure is represented in figure 1 , in comparison to the standard approach of finding voids using galaxies as tracers.

\section{A. Bayesian large-scale structure inference with the BORG algorithm}

This works builds upon previous results, obtained by the application of BORG (Bayesian Origin Reconstruction from Galaxies, BORG method) to SDSS main galaxy data (BORG SDSS). The BORG algorithm is a fully probabilistic inference machinery aiming at the analysis of linear and mildly-non-linear density and velocity fields in galaxy observations. It incorporates a physical model of cosmological structure formation, which translates the traditional task of reconstructing the non-linear three-dimensional density field into the task of inferring corresponding initial conditions from present cosmological observations. This approach yields a highly non-trivial Bayesian inference, requiring to explore very high-dimensional and non-linear spaces of possible solutions to the initial conditions problem from incomplete observations. Typically, these parameter spaces comprise on the order of $10^{6}$ to $10^{7}$ parameters, corresponding to the elements of the discretized observational domain.

Specifically, the BORG algorithm explores a posterior distribution consisting of a Gaussian prior, describing the statistical behavior of the initial density field at a 

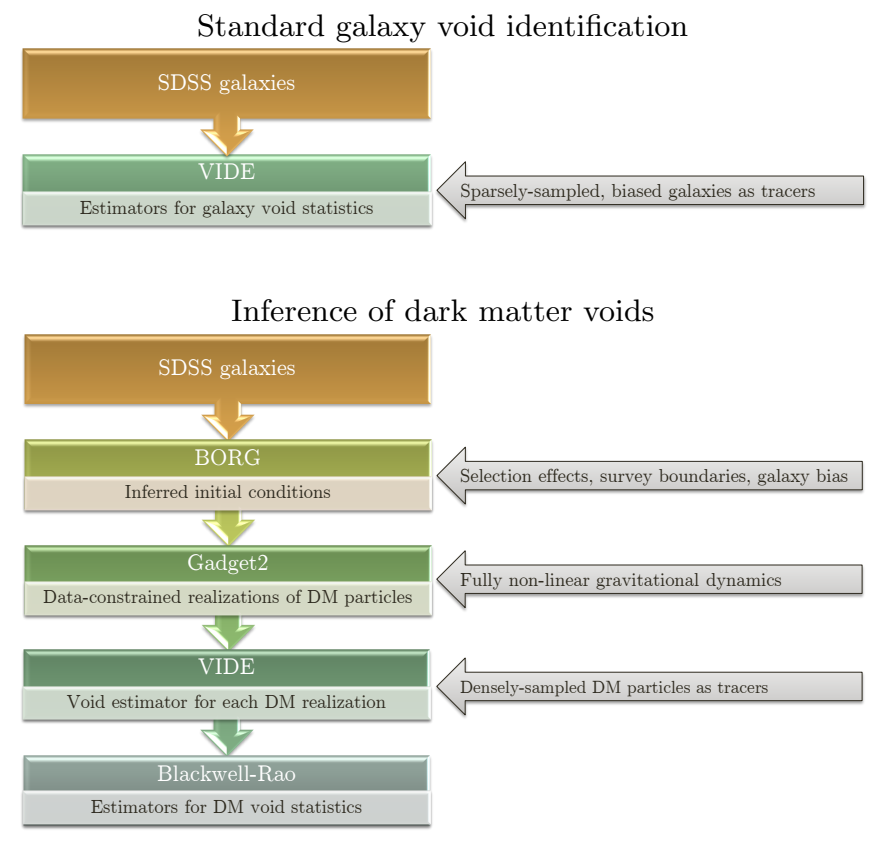

FIG. 1. Schematic representation of our methodology for the inference of dark matter voids (lower panel) in comparison to the standard approach for the identification of galaxy voids (upper panel).

cosmic scale factor of $a=10^{-3}$, linked via second-order Lagrangian perturbation theory (2LPT) to a Poissonian model of galaxy formation at the present epoch (for details see BORG method and BORG SDSS). As pointed out by previous authors (see e.g. Moutarde et al., 1991; Buchert, Melott \& Weiss, 1994; Bouchet et al., 1995; Scoccimarro, 2000; Bernardeau et al., 2002; Scoccimarro \& Sheth, 2002), 2LPT describes the one, two and three-point statistics correctly and represents higherorder statistics very well. Consequently, the BORG algorithm naturally accounts for features of the cosmic web, such as filaments, that are typically associated to highorder statistics induced by non-linear gravitational structure formation processes.

Besides physical structure formation, the posterior distribution also accounts for survey geometry, selection effects and noise, inherent to any cosmological observation. Corresponding full Bayesian uncertainty quantification is provided by exploring this highly non-Gaussian and non-linear posterior distribution via an efficient Hamiltonian Markov Chain Monte Carlo sampling algorithm (see BORG method for details). In order to account for luminosity dependent galaxy bias (Jasche \& Wandelt, 2013b) and to make use of automatic noise calibration, we further use modifications introduced to the original BORG algorithm by BORG SDSS.

In this work, we make use of the 12,000 samples of the posterior distribution generated by BORG SDSS, which constitute highly-detailed and accurate reconstructions of the initial and present-day density fields constrained by SDSS observations.

\section{B. Generation of data-constrained reconstructions}

We rely on a subset of statistically independent initial conditions realizations, provided by BORG SDSS, to generate a set of data-constrained realizations of the present large-scale structure. The initial density field, defined on a cubic equidistant grid with side length of $750 \mathrm{Mpc} / h$ and $256^{3}$ voxels, is populated by $512^{3}$ dark matter particles placed on a regular Lagrangian grid. The particles are evolved with 2LPT to the redshift of $z=69$, followed by a propagation with the non-linear GADGET- 2 cosmological code (Springel, Yoshida \& White, 2001; Springel, 2005) from $z=69$ to $z=0$. In this fashion, we generate fully non-linear, data-constrained reconstructions of the present-day large-scale dark matter distribution. For this work, we use an ensemble of 11 such reconstructions.

The final conditions inferred by BORG are accurate only at linear and mildly non-linear scales. Application of fully non-linear dynamics to the corresponding initial conditions acts as an additional filtering step, extrapolating predictions to unconstrained non-linear regimes. In a Bayesian approach, this new information can then be tested with complementary observations in the actual sky for updating our knowledge on the Universe.

An illustration ${ }^{1}$ of the non-linear filtering procedure is presented in figure 2. By comparing initial and final density fields, one can see correspondences between structures in the present Universe and their origins. Comparing the final density fields before and after filtering (middle and left panels), one can check the conformity of the linear and mildly non-linear structures at large and intermediate scales, correctly predicted by $2 \mathrm{LPT}$. Small-scale structures, corresponding to the deeply nonlinear regime, are much better represented after nonlinear filtering (resulting particularly in sharper filaments and clusters). $N$-body dynamics also resolves much more finely the substructure of voids - known to suffer from spurious artifacts in 2LPT, namely the presence of peaky, overdense spots where there should be deep voids (Neyrinck, 2013; Leclercq et al., 2013) - which is of relevance for the purpose of this work.

\section{Void finding and processing}

\section{Void finding}

We identify and post-process voids with the VIDE (Void IDentification and Examination) toolkit ${ }^{2}$ (VIDE), which uses a highly modified version of zOBOV (Neyrinck, 2008; Lavaux \& Wandelt, 2012; Sutter et al., 2012b) to create a Voronoi tessellation of the tracer particle population and the watershed transform to group Voronoi cells into

\footnotetext{
${ }^{1}$ In figures 2 and 3, we kept the coordinate system of BORG SDSS. 2 http://www.cosmicvoids.net
} 

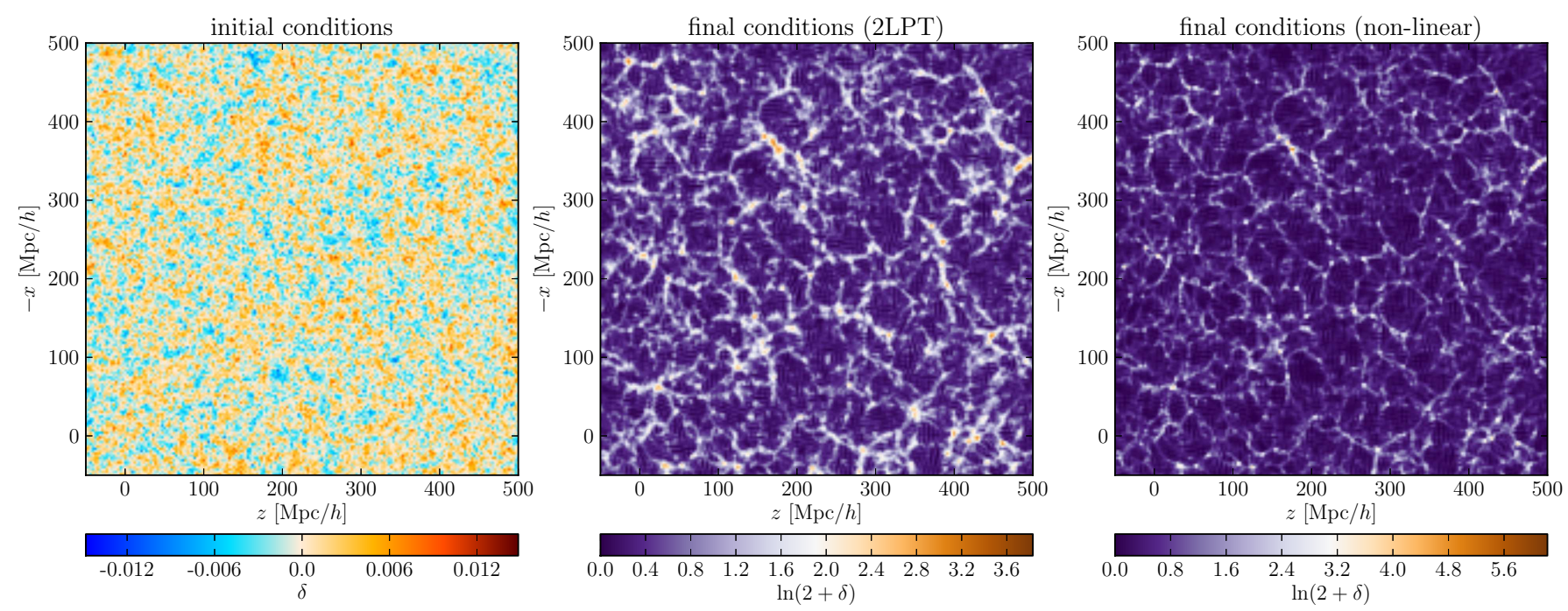

FIG. 2. Non-linear filtering of BORG results. Slices through one sample of initial (left panel) and final density fields (middle panel) inferred by BORG. The final density field (middle panel) is a prediction of the 2LPT model used by BORG. On the right panel, a slice through the data-constrained realization obtained with the same sample via non-linear filtering (fully non-linear gravitational structure formation starting from the same initial conditions) is shown.

zones and voids (Platen, van de Weygaert \& Jones, 2007). The watershed transform identifies catchment basins as the cores of voids, and ridgelines, which separate the flow of water, as the boundaries of voids. It naturally builds a nested hierarchy of voids (Lavaux \& Wandelt, 2012; Bos et al., 2012). For the purposes of this work, we examine all voids regardless of their position in the hierarchy. The pipeline imposes a density-based threshold within the void finding operation: voids only include as additional members Voronoi zones if the minimum ridge density between that zone and the void is less than 0.2 times the mean particle density (Platen, van de Weygaert \& Jones 2007; see Blumenthal et al. 1992; Sheth \& van de Weygaert 2004 for the role of the corresponding $\delta=-0.8$ underdensity). If a void consists of only a single zone (as they often do in sparse populations) then this restriction does not apply.

VIDE provides several useful definitions used in this work, such as the effective radius,

$$
R_{\mathrm{v}} \equiv\left(\frac{3}{4 \pi} V\right)^{1 / 3}
$$

where $V$ is the total volume of the Voronoi cells that contribute to the void. We use this radius definition to ignore voids with $R_{\mathrm{v}}$ below the mean particle spacing $\bar{n}^{-1 / 3}$ of the tracer population, as these are increasingly affected by Poisson fluctuations. VIDE also reports the volume-weighted center, or macrocenter, as

$$
\mathbf{x}_{\mathrm{v}} \equiv \frac{1}{\sum_{i} V_{i}} \sum_{i} \mathbf{x}_{i} V_{i}
$$

where $\mathbf{x}_{i}$ and $V_{i}$ are the positions and Voronoi volumes of each tracer particle $i$, respectively.
In each tracer population, the VIDE pipeline provides void estimators ; in particular, the three statistics we will focus on in section III: number count, ellipticity distribution and radial density profile.

In figure 3, we show slices through different dataconstrained realizations. The density of dark matter particles identified by VIDE as being part of a void is represented in gray scale. Note that, since zoBov essentially performs a division of space in different void regions with vanishingly-thin ridges, almost all particles initially present in the dark matter field are conserved. For clarity of the visualization, the quantity represented is $\ln (2+\delta)$ where $\delta$ is the density contrast of particles in voids. The SDSS galaxies used for the BORG analysis are overplotted as red dots. The core of dark matter voids (using a density threshold $\delta<-0.3$ ) is shown in color. As can be observed, dark matter voids also correspond to underdensities in the field traced by galaxies, which is in agreement with the results obtained by Sutter et al. (2014b) in simulations.

\section{Selection of voids}

The VIDE pipeline identifies all dark matter voids in the non-linear data-constrained realizations described in section II B. These live in boxes of $750 \mathrm{Mpc} / h$ side length with periodic boundary conditions. In order to select physically meaningful dark matter void candidates, we have to select a subsample of voids which intersect the volume of the box actually constrained by SDSS galaxies.

As described in BORG SDSS, unobserved and observed regions in the inferred final density fields do not appear visually distinct, a consequence of the excellent perfor- 

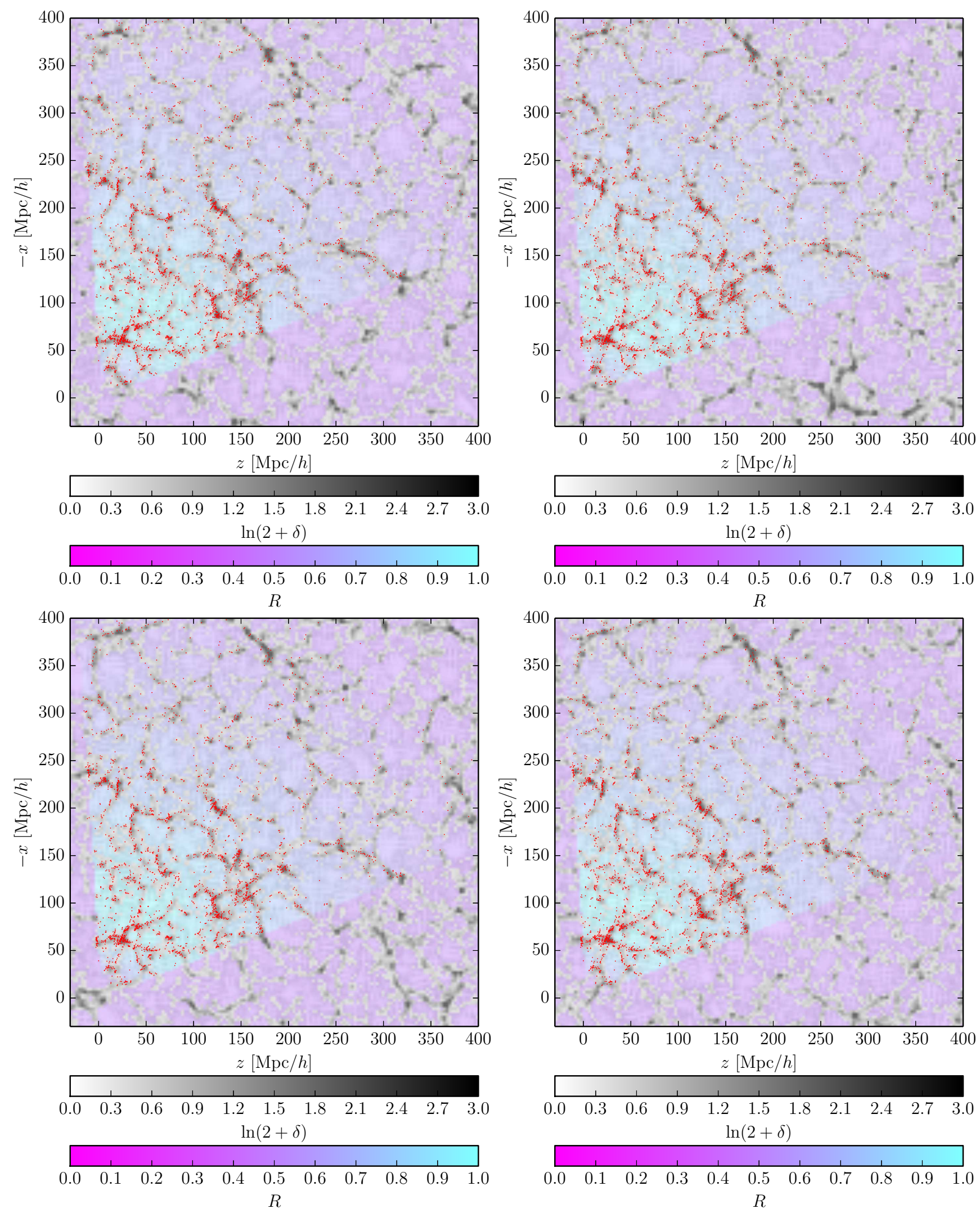

FIG. 3. Slices through different data-constrained realizations used to build samples of the dark matter void catalog. The SDSS galaxies used for the inference with BORG are represented as red dots. The density of dark matter particles identified by VIDE as being part of a void is shown in gray scale. In color, we show the particles that live in the core of dark matter voids (in a density environment smaller than -0.3 times the average density). The survey response operator $R$ shows how well the results are constrained by the data (see text for details). In the observed region, the data are strongly informative about the cosmic web in general and voids in particular; the reconstructions are not prior-dominated. 
mance of the 2LPT model implemented in BORG as a physical description of structure formation. In addition, due to the non-local transport of observational information between initial and final conditions, the region influenced by data extends beyond the survey boundaries and the large-scale structure appears continuous there. The fact that data constraints can radiate out of the survey volume has been known since the first constrained reconstructions of the mass distribution (Bertschinger, 1987; Hoffman \& Ribak, 1991; van de Weygaert \& Bertschinger, 1996), where a power spectrum prior was assumed to sample constrained Gaussian random fields. Here, as detailed in BORG SDSS, constraints are propagated by the structure formation model assumed in the inference process (2LPT), which accounts not only for two-point statistics, but for the full hierarchy of correlators, in its regime of validity. Therefore, dark matter voids candidates intersecting the survey boundaries can be considered as physical if a significant fraction of their volume is influenced by the data.

The survey response operator $R$ is a voxel-wise function representing simultaneously the survey geometry (observed and unobserved regions) and the selection effects in galaxy catalogs. Here, we kept for $R$ the average over the six luminosity bins used in the BORG SDSS run (for details see BORG SDSS). For the purpose of this work, we keep all void candidates whose center is in a region where $R$ is strictly positive. This region represents $7.9 \times 10^{7} \mathrm{cubic} \mathrm{Mpc} / h$, around $18.7 \%$ of the full box. In each of the 11 realizations used in this work, we kept around 166,000 data-constrained voids out of 886,000 voids in the entire box.

In figure 3 , the survey response operator is shown in color from purple (totally unobserved region) to blue (region fully constrained by the data). One can see the correct propagation of information operated by BORG, as voids appear continuous at the survey boundaries.

\section{Blackwell-Rao estimators for dark matter void realizations}

A particular advantage of our Bayesian methodology is the ability to provide accurate uncertainty quantification for derived dark matter void properties. In particular, the Markovian samples provided by BORG SDSS permit us to employ a Blackwell-Rao estimator to describe the posterior distribution for inferred dark matter voids. Specifically, we are interested in deriving the posterior distribution $\mathcal{P}(x \mid d)$ of a dark matter void property $x$ given observations $d$. Using the realizations of the initial conditions $\delta^{\mathrm{i}}$ taken from BORG SDSS and the dark matter void realizations $V$ generated by the approach described in sections II B and II C, we obtain

$$
\begin{aligned}
\mathcal{P}(x \mid d) & =\int \mathcal{P}(x \mid V) \mathcal{P}\left(V, \delta^{\mathrm{i}} \mid d\right) \mathrm{d} V \mathrm{~d} \delta^{\mathrm{i}} \\
& =\int \mathcal{P}(x \mid V) \mathcal{P}\left(V \mid \delta^{\mathrm{i}}, d\right) \mathcal{P}\left(\delta^{\mathrm{i}} \mid d\right) \mathrm{d} V \mathrm{~d} \delta^{\mathrm{i}} \\
& =\int \mathcal{P}(x \mid V) \delta^{\mathrm{D}}\left(V-\tilde{V}\left(\delta^{\mathrm{i}}\right)\right) \mathcal{P}\left(\delta^{\mathrm{i}} \mid d\right) \mathrm{d} V \mathrm{~d} \delta^{\mathrm{i}} \\
& =\int \mathcal{P}\left(x \mid \tilde{V}\left(\delta^{\mathrm{i}}\right)\right) \mathcal{P}\left(\delta^{\mathrm{i}} \mid d\right) \mathrm{d} \delta^{\mathrm{i}} \\
& \approx \frac{1}{N} \sum_{k} \mathcal{P}\left(x \mid \tilde{V}\left(\delta_{k}^{\mathrm{i}}\right)\right) \\
& =\frac{1}{N} \sum_{k} \mathcal{P}\left(x \mid V_{k}\right)
\end{aligned}
$$

where we assumed the dark matter void templates $V$ to be conditionally independent of the data $d$ given the initial conditions $\delta^{\mathrm{i}}$, and to derive uniquely from the initial density field via the procedure described in sections II B and II C, yielding $\mathcal{P}\left(V \mid \delta^{\mathrm{i}}, d\right)=\mathcal{P}\left(V \mid \delta^{\mathrm{i}}\right)=\delta^{\mathrm{D}}\left(V-\tilde{V}\left(\delta^{\mathrm{i}}\right)\right)$. We also exploited the fact that we have a sampled representation of the initial conditions posterior distribution $\mathcal{P}\left(\delta^{\mathrm{i}} \mid d\right) \approx 1 / N \sum_{k} \delta^{\mathrm{D}}\left(\delta^{\mathrm{i}}-\delta_{k}^{\mathrm{i}}\right)$, where $k$ labels one of the $N$ samples. The last line of equation (3) represents the Blackwell-Rao estimator for void property $x$ to be inferred from our dark matter void catalogs $V_{k}$, providing thorough Bayesian means to quantify uncertainties. It consists of a mixture distribution over different realizations of dark matter void templates.

The VIDE pipeline provides estimated means and variances for derived quantities $x$, allowing us to model the distributions $\mathcal{P}\left(x \mid V_{k}\right)$ as Gaussians with mean $x_{k}$ and variance $\sigma_{k}^{2}$, for respective dark matter void templates. The final expression for the posterior distribution of $x$ given the data is therefore

$$
\mathcal{P}(x \mid d) \approx \frac{1}{N} \sum_{k} \frac{1}{\sqrt{2 \pi \sigma_{k}^{2}}} \exp \left(-\frac{1}{2} \frac{\left(x-x_{k}\right)^{2}}{\sigma_{k}^{2}}\right) .
$$

Even though we have access to non-Gaussian uncertainty quantification via the posterior distribution given in equation (4), for the presentation in this paper we will be content with estimating means and variances. The mean for $x$ given $d$ is

$$
\langle x \mid d\rangle \approx \frac{1}{N} \sum_{k} x_{k}
$$

and the variance is

$$
\left\langle(x-\langle x\rangle)^{2} \mid d\right\rangle \approx \frac{1}{N} \sum_{k}\left(x_{k}^{2}+\sigma_{k}^{2}\right)-\langle x \mid d\rangle^{2} .
$$

As described in section IIC 2, we select voids in the data-constrained regions of reconstructions of the dark matter density field. Since these regions are the same in different reconstructions, the different void catalogs 
describe the same region of the actual Universe. For this reason, while estimating uncertainties, it is not possible to simply use all the voids in our catalogs as if they were independent $^{3}$. However, using an increasing number of reconstructions, we shall still see a decrease of statistical uncertainty. Indeed, from (5) and (6) it follows that

$$
\left\langle(x-\langle x\rangle)^{2} \mid d\right\rangle \leq \frac{1}{N} \sum_{k} \sigma_{k}^{2},
$$

which means that the combination of different realizations will generally yield an improved estimator for any original statistics.

Note that this procedure is completely general and applies to any estimator provided by the VIDE pipeline.

\section{E. Void catalogs for comparison of our results}

In section III, we will compare our results for dark matter voids to state-of-the-art results for galaxy voids. To do so, we use the catalogs of Sutter et al. (2012b) based on the SDSS DR7 galaxies, publicly available at http://www.cosmicvoids.net. In particular, we compare to the voids found in the bright1 and dim1 volumelimited galaxy catalogs, for which the mean galaxy separations are 8 and $3 \mathrm{Mpc} / h$, respectively (for details, see Sutter et al., 2012b).

Assessment of our results for dark matter voids in SDSS data also require systematic comparison to dark matter voids found in cosmological simulations. We ran 11 such unconstrained simulations with the same setup as described in section II B for the generation of dataconstrained realizations. We started from Gaussian random fields with an Eisenstein \& Hu (1998, 1999) power spectrum using the fiducial cosmological parameters of the BORG analysis $\left(\Omega_{\mathrm{m}}=0.272, \Omega_{\Lambda}=0.728\right.$, $\Omega_{\mathrm{b}}=0.045, h=0.702, \sigma_{8}=0.807, n_{\mathrm{s}}=0.961$, see BORG SDSS). These initial density fields, defined in a $750 \mathrm{Mpc} / h$ cubic box of $256^{3}$ voxels, are occupied by a Lagrangian lattice of $512^{3}$ dark matter particles. These are evolved to $z=69$ with $2 \mathrm{LPT}$ and from $z=69$ to $z=0$ with GADGET-2. As for constrained realizations, in our simulations we selected the voids located inside the observed SDSS volume (see section II C 2) and combined properties using Blackwell-Rao estimators (see section IID).

\section{PROPERTIES OF DARK MATTER VOIDS}

In this section, we describe the statistical properties of the dark matter voids found in the data-constrained parts

\footnotetext{
3 We generally recommend special care for proper statistical treatment while working with the data-constrained realizations of our dark matter void catalog, especially if one wants to use frequentist estimators of void properties.
}

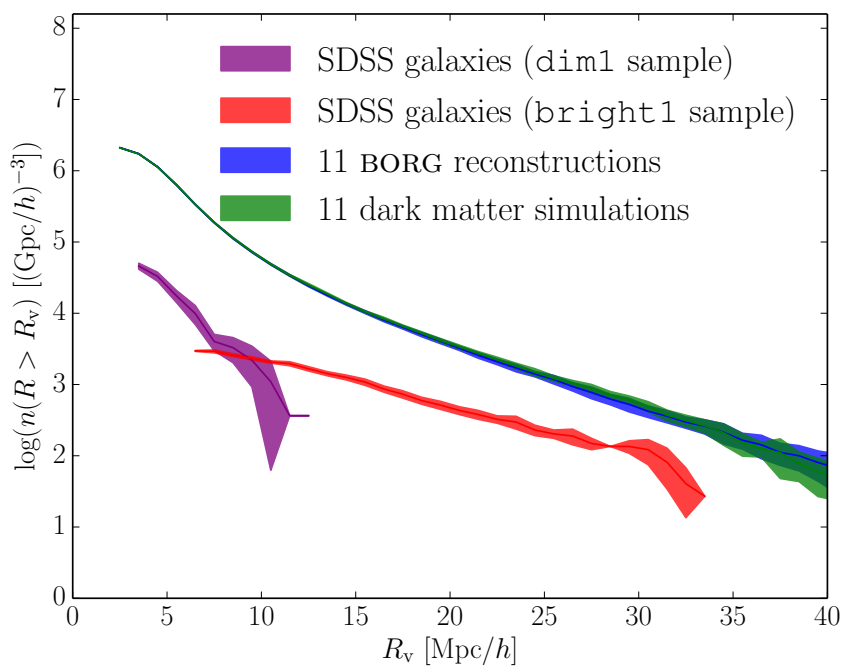

FIG. 4. Cumulative void number functions. The results from 11 BORG reconstructions (blue) are compared to a dark matter $N$-body simulation (green) and to the galaxy voids directly found in two volume-limited sub-samples of the SDSS DR7 (dim1, purple and bright1, red). The solid lines are the measured or predicted number functions and the shaded regions are the 2- $\sigma$ Poisson uncertainties. Fewer voids are found in observations than in dark matter simulations, due to the sparsity and bias of tracers, as well as observational uncertainty coming from the survey geometry and selection effects. Number functions from BORG reconstructions agree with simulations at all scales.

of our reconstructions of the SDSS volume. We focus on three key statistical summaries abundantly described in the literature: number count, ellipticity distribution and radial density profiles.

\section{A. Number function}

The number function of voids provides a simple, easily accessible, and surprisingly sensitive cosmological probe. For example, the number function has been shown to respond to coupled dark matter-dark energy (Li \& Zhao, 2009; Sutter et al., 2015a), modified gravity (Li, Zhao \& Koyama, 2012; Clampitt, Cai \& Li, 2013), and variations in fundamental cosmological parameters (Pisani et al., 2015). While most studies of the number function take place in $N$-body simulations, there has also been significant theoretical and analytical work, beginning with the excursion set formulation of Sheth \& van de Weygaert (2004) and continuing through further enhancements to account for the complex nature of void shapes (Jennings, Li \& Hu, 2013). As previous authors (Müller et al., 2000; Sutter et al., 2012b, 2014c; Nadathur \& Hotchkiss, 2014; Nadathur et al., 2014) have noted, there tend to be fewer voids in observations than in numerical simulations, especially for small voids. This is due to the conjugate effects of sparsity and biasing of tracers, which can mod- 
ify the number function in complex ways (Furlanetto \& Piran, 2006; Sutter et al., 2014a,c), as well as survey geometries and selection effects, which can non-trivially diminish the void population. However, recently Sutter et al. (2014c) showed a correspondence between observed and theoretical number functions once these factors are taken into account.

Figure 4 shows the cumulative void number function in BORG reconstructions (blue) compared to dark matter simulations using the same setup (green) and to galaxy voids in the SDSS DR7 (red and purple). The confidence regions are 2- $\sigma$ Poisson uncertainties and the blue and green lines use Blackwell-Rao estimators to combine the results in 11 realizations.

We can immediately note the excellent agreement between simulations and dark matter voids candidates in the SDSS as found by our methodology. The two void populations are almost indistinguishable at all scales, which demonstrates that the data-constrained number function predicted by our methodology is exactly that of dark matter voids in numerical simulations. In particular, this proves that our framework correctly permits to circumvent the effects of sparsity and biasing of SDSS galaxies on void number count. Indeed, dark matter voids in our reconstructions are denselysampled with the same number density as in simulations, $\bar{n}=0.318(\mathrm{Mpc} / h)^{-3}\left(512^{3}\right.$ particles in $\left.(750 \mathrm{Mpc} / h)^{3}\right)$ compared to $\bar{n} \approx 10^{-3}(\mathrm{Mpc} / h)^{-3}$ for SDSS galaxies (Sutter et al., 2012b). Furthermore, any incorrect treatment of galaxy bias by the BORG algorithm would result in a residual bias in our reconstructions that would yield an erroneous void number function as compared to simulations (Sutter et al., 2014a). The absence of any such feature confirms that galaxy bias is correctly accounted for in our analysis and further validates the framework described in BORG SDSS.

Additionally, due to the high density of tracer particles, we find at least around one order of magnitude more voids at all scales than the voids directly traced by the SDSS galaxies, which sample the underlying mass distribution only sparsely. This results in a drastic reduction of statistical uncertainty in void catalogs, as we demonstrate in sections III B and III C.

\section{B. Ellipticity distribution}

The shape distribution of voids is complementary to overdense probes of the dark matter density field such as galaxy clusters. Indeed, as matter collapses to form galaxies, voids expand and can do so aspherically. While Icke (1984) argued that voids are expected to become more spherical as they expand, Platen, van de Weygaert \& Jones (2008) found that the shape distribution of voids remains complex at late times and showed that the aspherical expansion of voids is strongly linked to the ex-

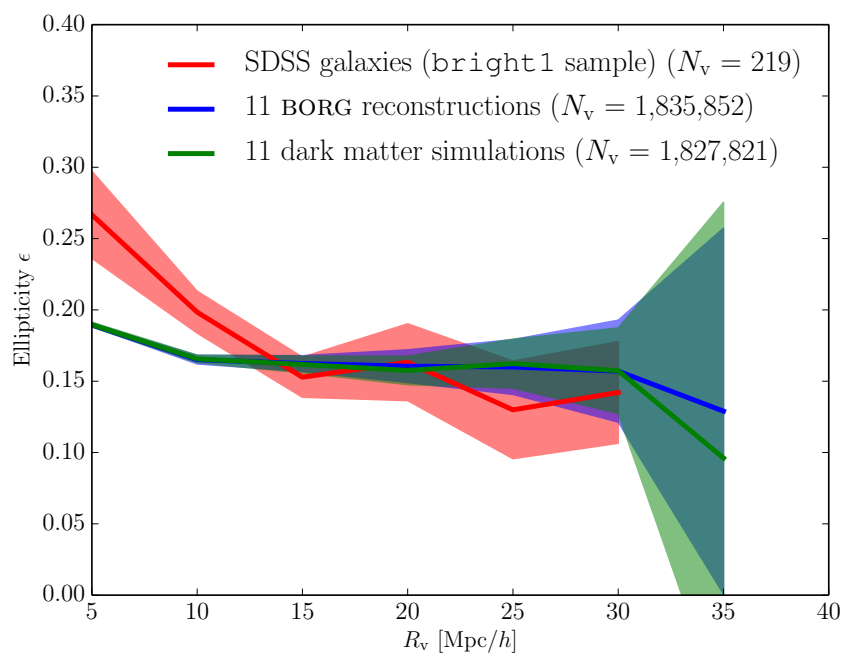

FIG. 5. Distribution of ellipticities $\epsilon$ versus effective radii of voids. The solid line shows the mean, and the shaded region is the $2-\sigma$ confidence region estimated from the standard error on the mean in each radial bin. Small galaxy voids are found more elliptical than dark matter voids because of important Poisson fluctuations below the mean galaxy separation $(8 \mathrm{Mpc} / h)$. Ellipticities of dark matter voids in BORG reconstructions and simulations agree at all scales, and the statistical uncertainty in their determination is drastically reduced in comparison to galaxy void catalogs.

ternal tidal influence ${ }^{4}$. Therefore, the shapes of empty regions generally change during cosmic evolution and retain information on their formation history. In particular, the void shape distribution potentially serves as a powerful tracer of the equation of state of dark energy (Lee \& Park, 2006; Park \& Lee, 2007; Biswas, Alizadeh \& Wandelt, 2010; Lavaux \& Wandelt, 2012; Bos et al., 2012). In addition, the mean stretch of voids along the line of sight may be used for an application of the AlcockPaczynski test (Alcock \& Paczynski, 1979; Ryden, 1995; Lavaux \& Wandelt, 2012; Sutter et al., 2012a, 2014d; Hamaus et al., 2014a).

For these applications, it is of crucial importance for the void catalog to be unaffected by systematics due to baryonic physics. Furthermore, as pointed out by Bos et al. (2012), in sparse populations such as galaxies it is very difficult to statistically separate $\Lambda$ CDM from alternative cosmologies using void shapes. As we now show, our framework allows to access void shapes at the level of the dark matter distribution, deeper than with the galaxies, and to reduce the statistical uncertainty due to their sparsity. Note that all the phase information and spatial organization of the LSS is unaffected by our prior assumptions, which generally affect the density amplitudes

\footnotetext{
4 Tidal effects are taken into account in our analysis since BORG models gravitational evolution up to second order in Lagrangian perturbation theory.
} 
via the cosmological power spectrum. The geometry of voids discussed here is therefore strongly constrained by the observations.

We simplify the discussion by focusing on the ellipticity, computed by the VIDE toolkit using the eigenvalues of the inertia tensor (for details, see VIDE). Figure 5 shows the mean ellipticity and the standard error on the mean (i.e. $\sigma / \sqrt{N_{\mathrm{v}}}$, where $\sigma$ is the standard deviation and $N_{\mathrm{v}}$ is the number of voids) as a function of void effective radius. The red line represents the galaxy voids directly found in the SDSS data, the blue line the dark matter voids of our data-constrained catalogs, and the green line the voids found in dark matter simulations prepared with the same setup. The blue and green lines use Blackwell-Rao estimators to combine the results of 11 realizations. For the interpretation of the ellipticity of small galaxy voids, it is useful to recall that the mean galaxy separation in the bright 1 sample is $8 \mathrm{Mpc} / h$, meaning that Poisson fluctuations will be of importance for voids whose effective radius is below this scale.

The comparison between dark matter voids of BORG reconstructions and of simulations shows that the predicted ellipticities fully agree with the expectations at all scales. This further demonstrates that our candidates qualify as dark matter voids as defined by numerical simulations, in particular alleviating the galaxy bias problem. Furthermore, as already noted, our inference framework produces many more voids than sparse galaxy catalogs, especially at small scales. This results in a radical reduction of statistical uncertainty in the ellipticity prediction for small dark matter voids as compared to galaxy voids, as can be observed in figure 5 .

\section{Radial density profiles}

The radial density profile of voids, reconstructed in real space using techniques such as those described in Pisani et al. (2014), can be used to test general relativity and constrain dynamical dark energy models (Shoji \& Lee, 2012; Spolyar, Sahlén \& Silk, 2013). More generally, it shows a self-similar structure (Colberg et al., 2005; Ricciardelli, Quilis \& Varela, 2014; Hamaus, Sutter \& Wandelt, 2014; Nadathur et al., 2014), and characterizes the LSS in a fundamental way (van de Weygaert \& van Kampen, 1993). All results presented in this section assume that dark matter particles in BORG reconstructions and in simulations live in physical space. The BORG algorithm automatically mitigates redshift space distortions by treating anisotropic features in the data as noise (BORG SDSS). Furthermore, as pointed out by Padilla, Ceccarelli \& Lambas (2005), redshift space distortions have very mild effects on void density profiles. We therefore expect our results to be robust under the transformation from real to redshift space.

Using VIDE, we construct the one-dimensional radial density profiles of stacked voids for various void sizes. Note that we do not apply any rescaling to the void sizes as we stack. Figure 6 shows two such profiles, for voids of effective radius in the range $6-8 \mathrm{Mpc} / h$ (left panel) and $20-25 \mathrm{Mpc} / h$ (right panel). The solid lines show the mean and the shaded regions are the $2-\sigma$ confidence regions estimated from the standard error on the mean, using Blackwell-Rao estimators for BORG reconstructions and dark matter simulations. At the level of statistical error in our results, our reconstructions show radial density profiles in agreement with simulations at all radii and for all void sizes. Note that, if small voids essentially reflect the prior information used for the BORG analysis and $N$-body filtering, bigger voids are strongly constrained by the data. The profile shapes agree nicely with the results of Sutter et al. (2014a); Hamaus, Sutter \& Wandelt (2014) from dark matter simulations: higher ridges and lower central densities in smaller voids. Specifically, our reconstructions exhibit the same behaviour as simulations, with a transition scale between small overcompensated to large undercompensated voids (Ceccarelli et al., 2013; Paz et al., 2013; Cai et al., 2014; Hamaus et al., 2014b).

In contrast, galaxy void profiles at the same scales are strongly noise-dominated. This is due to the sparsity and biasing of galaxies, which are alleviated with the present approach. In particular, our methodology performs a meaningful compromise between data and prior information, which predicts corrected shapes and smaller variance for the profiles of dark matter voids as compared to galaxy voids. Note that at the same physical scales (e.g. $20 \mathrm{Mpc} / h$ ), galaxy voids and dark matter voids have different ridge heights. This is because a deeper void hierarchy emerges in higher tracer sampling densities, affecting the compensation of voids at a given size (Sutter et al., 2014a).

In addition to the location of all dark matter particles, our inference framework also provides their individual velocity vectors, which are predicted from gravitational clustering. While the direct measurement of individual galaxy velocities is very difficult in most observations, our reconstruction technique readily allows to infer the velocity profile of voids. This allows to make a connection between a static (based on the density profiles) and a dynamic (based on the velocity profiles) characterization of voids. In particular, as mentioned before, our results agree with the existence of a transition scale between two regimes: undercompensated, inflowing voids and overcompensated, outflowing voids, respectively known as void-in-cloud and void-in-void in the terminology originally introduced by Sheth \& van de Weygaert (2004).

\section{SUMMARY AND CONCLUSIONS}

This paper is an example of the rich variety of scientific results that have been produced by the recent application (BORG SDSS) of the Bayesian inference framework BORG (BORG method) to the Sloan Digital Sky Survey main sample galaxies. We proposed a method designed to find 

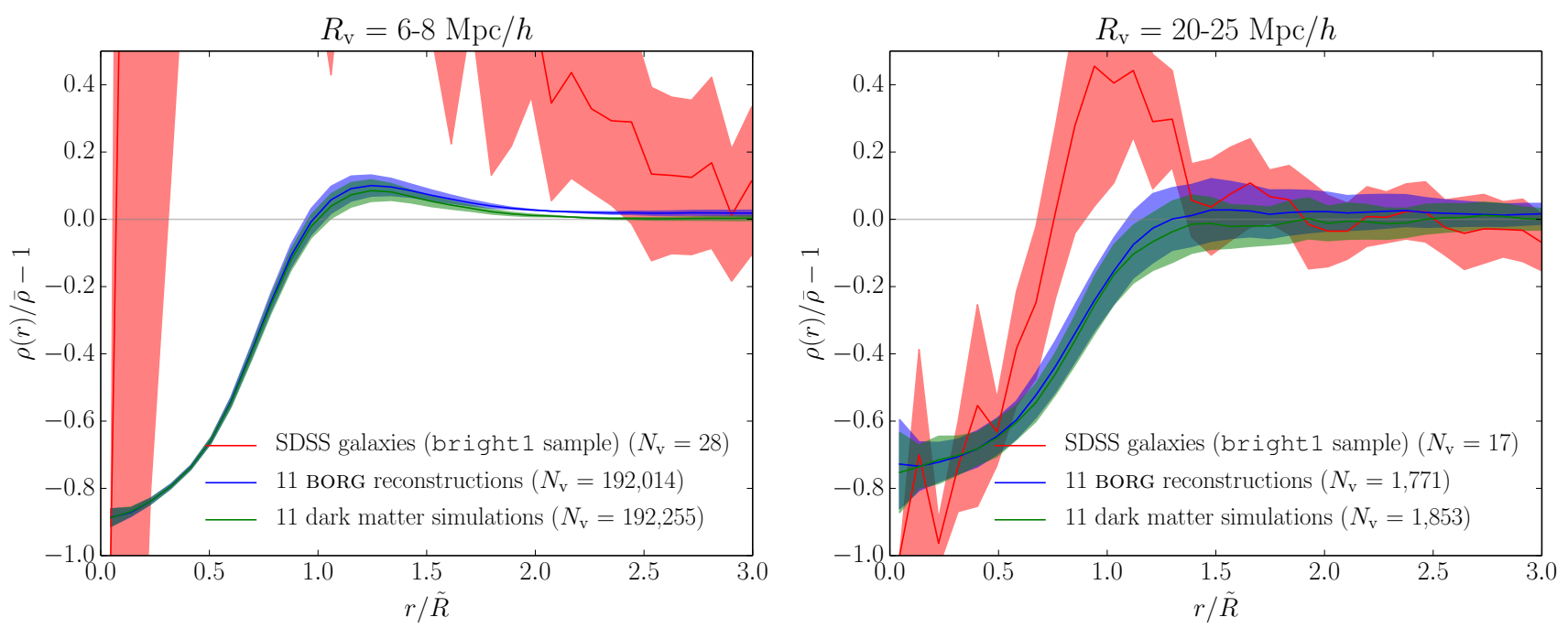

FIG. 6. One-dimensional radial density profiles of stacked voids, for voids of effective radius in the range 6-8 Mpc/ $h$ (left) and $20-25 \mathrm{Mpc} / h$ (right). $\tilde{R}$ corresponds to the median void size in the stack. The solid line shows the mean, and the shaded region is the $2-\sigma$ confidence region estimated from the standard error on the mean in each radial bin. Galaxy void profiles are strongly noise-dominated, contrary to dark matter voids. The heights of compensation ridges are different because dark matter voids are identified in a higher density of tracers, which induces a deeper void hierarchy.

dark matter void candidates in the Sloan volume. In doing so, we proved that physical voids in the dark matter distribution can be correctly identified by the ab initio analysis of galaxy surveys.

Our method relies on the physical inference of the initial conditions for the entire LSS (BORG method; BORG SDSS). Starting from these, we generated realizations of the LSS using a fully non-linear cosmological code. In this fashion, as described in section IIB, we obtained a set of data-constrained reconstructions of the presentday dark matter distribution. The use of fully non-linear dynamics as a filter allowed us to extrapolate the predictions of BORG to the unconstrained non-linear regimes and to obtain an accurate description of structures. As described in section II C, we identified the voids in these reconstructions using the void finder of the VIDE pipeline (VIDE) and applied an additional selection criterion to limit the final catalogs of dark matter voids candidates to regions covered by observations. To check that these candidates qualify for physical voids, we analyzed our catalogs in terms of a set of statistical diagnostics. We focused on three key void statistics, well understood both in data and in simulations, provided by the VIDE toolkit: number function, ellipticity distribution and radial density profile. As mentioned in section II E, for comparison of our results, we used the void catalog of Sutter et al. (2012b), directly based on SDSS main sample galaxies, and unconstrained dark matter simulations produced with the same setup as our reconstructions.

For quantifying the uncertainty, we adopted the same Bayesian philosophy as in the LSS inference framework: several void catalogs are produced, based on different samples of the BORG posterior probability distribution function. Each of them represents a realization of the actual dark matter voids in the Sloan volume, and the variation between these catalogs quantifies the remaining uncertainties of various sources (in particular, survey geometry and selection effects, see BORG SDSS for a complete discussion). In order to produce a statistically meaningful combination of our different dark matter void catalogs, in section II D, we introduced Blackwell-Rao estimators. We showed that the combination of different realizations generally yields an improved estimator for any original void statistic.

For all usual void statistics (number function in section III A, ellipticity distribution in section III B and radial density profiles in section IIIC), we found remarkably good agreement between predictions for dark matter voids in our reconstructions and expectations from numerical simulations. This validates our inference framework and qualifies the candidates to physically reasonable dark matter voids, probing a level deeper in the mass distribution hierarchy than galaxies. Further, since sparsity and biasing of tracers modify these statistics (Sutter et al., 2014a), it means that these effects have been correctly accounted for in our analysis. Indeed, in BORG SDSS we showed that BORG accurately accounts for luminosity-dependent galaxy bias and performs automatic calibration of the noise level within a fully Bayesian approach. Building on the detailed representation of initial density fields, our reconstructions possess a high density of tracers, $\bar{n}=0.318(\mathrm{Mpc} / h)^{-3}$, contrary to galaxies, which sample the underlying mass distribution only sparsely $\left(\bar{n} \approx 10^{-3}(\mathrm{Mpc} / h)^{-3}\right)$.

Another important aspect of our methodology is that the use of full-scale physical density fields instead of a 
scarce population of galaxies allows to adjust the density of tracers to reduce shot-noise at the desired level. In our analysis, we found at least one order of magnitude more voids at all scales. This yields a radical reduction of statistical uncertainty in noise-dominated void catalogs, as we have shown for ellipticity distributions and density profiles.

In summary, our methodology permits to alleviate the issues due to the conjugate and intricate effects of sparsity and biasing on galaxy void catalogs, to drastically reduce statistical uncertainty in void statistics, and yields new catalogs of dark matter voids for a variety of cosmological applications. For example, these enhanced data sets can be used for cross-correlation with other cosmological probes such as the cosmic microwave background, to study the integrated Sachs-Wolfe effect, or gravitational lensing shear maps. Along with the ensemble mean density field and corresponding standard deviations inferred by BORG, published as supplementary material with (BORG SDSS), we believe that the catalogs of our dark matter voids candidates in the Sloan volume can be of interest to the scientific community. For this reason, all the void catalogs used to produce the results described in this paper will be made publicly available at http://www.cosmicvoids.net.

Our Bayesian methodology, based on inference with BORG and subsequent non-linear filtering of the results, assumes some prior information, namely the standard $\Lambda$ CDM cosmological framework and initially Gaussian density fluctuations. We want to emphasize that any analysis using our constrained catalogs will be biased toward the confirmation of these assumptions. Therefore, this method will be only applicable if the data contain sufficient support for the presence of non-standard cosmology to overrule the preference for $\Lambda$ CDM and Gaussianity in our prior. However, any significant departure from standard cosmology means that the prior has been overridden by the likelihood and that such deviations really are supported by the data.

While the recommendations of Sutter et al. (2014a) for quantifying and disentangling the effects of sparsity and biasing depend on specific survey details, our inference framework is extremely general. It allows to translate void statistics from current and future galaxy surveys to theory-like, high-resolution dark matter predictions. In this fashion, it is straightforward to decide if any particular void statistic can be directly informative about cosmology. These results indicate a new promising path towards effective and precise void cosmology at the level of the dark matter field.

\section{ACKNOWLEDGMENTS}

We thank Guilhem Lavaux and Alice Pisani for valuable discussions and help with the VIDE pipeline, and all participants in the "Cosmic Voids in the Next Generation of Galaxy Surveys" Workshop held in Columbus, Ohio, for insightful comments. We are grateful to the referee for a very constructive report which helped finalizing this work. Special thanks go to Stéphane Rouberol for his support, in particular for guaranteeing flawless use of all required computational resources. The results described in this paper have been presented at the 49th "Rencontres de Moriond" Cosmology Session and at the International Astronomical Union Symposium 306 "Statistical Challenges in 21st Century Cosmology". We would like to express our gratitude to the organizers. FL acknowledges funding from an AMX grant (École polytechnique ParisTech). JJ is partially supported by a Feodor Lynen Fellowship by the Alexander von Humboldt foundation and BW's Chaire d'Excellence from the Agence Nationale de la Recherche (ANR-10-CEXC-004-01). PMS is supported by the INFN IS PD51 "Indark". This work has been done within the Labex ILP (reference ANR10-LABX-63) part of the Idex SUPER, and received financial state aid managed by the Agence Nationale de la Recherche, as part of the programme Investissements d'avenir under the reference ANR-11-IDEX-0004-02.

(Abel, Hahn \& Kaehler, 2012) T. Abel, O. Hahn, R. Kaehler, Tracing the dark matter sheet in phase space, Mon. Not. R. Astron. Soc. 427, 61 (2012), arXiv:1111.3944 [astro-ph.CO].

(Alcock \& Paczynski, 1979) C. Alcock, B. Paczynski, An evolution free test for non-zero cosmological constant, Nature 281, 358 (1979).

(Aragon-Calvo \& Szalay, 2013) M. A. Aragon-Calvo, A. S. Szalay, The hierarchical structure and dynamics of voids, Mon. Not. R. Astron. Soc. 428, 3409 (2013), arXiv:1203.0248 [astro-ph.CO].

(Benson et al., 2003) A. J. Benson, F. Hoyle, F. Torres, M. S. Vogeley, Galaxy voids in cold dark matter universes, Mon. Not. R. Astron. Soc. 340, 160 (2003), astro-ph/0208257.

(Bernardeau et al., 2002) F. Bernardeau, S. Colombi, E. Gaztañaga, R. Scoccimarro, Large-scale structure of the Universe and cosmological perturbation theory, Physics Reports 367, 1 (2002), astro-ph/0112551.

(Bertschinger, 1987) E. Bertschinger, Path integral methods for primordial density perturbations - Sampling of constrained Gaussian random fields, Astrophys. J. Lett. 323, L103 (1987).

(Betancort-Rijo et al., 2009) J. Betancort-Rijo, S. G. Patiri, F. Prada, A. E. Romano, The statistics of voids as a tool to constrain cosmological parameters: $\sigma_{8}$ and $\Gamma$, Mon. Not. R. Astron. Soc. 400, 1835 (2009), arXiv:0901.1609.

(Biswas, Alizadeh \& Wandelt, 2010) R. Biswas, E. Alizadeh, B. D. Wandelt, Voids as a precision probe of dark energy, Phys. Rev. D 82, 023002 (2010), arXiv:1002.0014 [astro-ph.CO].

(Blumenthal et al., 1992) G. R. Blumenthal, L. N. da Costa, D. S. Goldwirth, M. Lecar, T. Piran, The largest possible voids, Astrophys. J. 388, 234 (1992).

(Bos et al., 2012) E. G. P. Bos, R. van de Weygaert, K. Dolag, V. Pettorino, The darkness that shaped the void: dark energy and cosmic voids, Mon. Not. R. Astron. Soc. 426, 440 (2012), arXiv:1205.4238 [astro-ph.CO].

(Bouchet et al., 1995) F. R. Bouchet, S. Colombi, E. Hivon, R. Juszkiewicz, Perturbative Lagrangian approach to gravitational instability., Astron. \& Astrophys. 296, 575 (1995).

(Buchert, Melott \& Weiss, 1994) T. Buchert, A. L. Melott, A. G. Weiss, Testing Higher-Order Lagrangian Perturbation Theory Against Numerical Simulations - 1. Pancake Models, Astron. \& Astrophys. 288, 349 (1994), astro-ph/9309056.

(Cai et al., 2014) Y.-C. Cai, M. C. Neyrinck, I. Szapudi, S. Cole, C. S. Frenk, A Possible Cold Imprint of Voids on the Microwave Background Radiation, Astrophys. J. 786, 110 (2014), arXiv:1301.6136 [astro-ph.CO]. 
(Ceccarelli et al., 2013) L. Ceccarelli, D. Paz, M. Lares, N. Padilla, D. G. Lambas, Clues on void evolution - I. Large-scale galaxy distributions around voids, Mon. Not. R. Astron. Soc. 434, 1435 (2013), arXiv:1306.5798 [astro-ph.CO].

(Clampitt, Cai \& Li, 2013) J. Clampitt, Y.-C. Cai, B. Li, Voids in modified gravity: excursion set predictions, Mon. Not. R. Astron. Soc. 431, 749 (2013), arXiv:1212.2216 [astro-ph.CO].

(Clampitt \& Jain, 2014) J. Clampitt, B. Jain, Lensing Measurements of the Mass Distribution in SDSS Voids, ArXiv e-prints (2014), arXiv:1404.1834.

(Colberg et al., 2005) J. M. Colberg, R. K. Sheth, A. Diaferio, L. Gao, N. Yoshida, Voids in a $\Lambda C D M$ universe, Mon. Not. R. Astron. Soc. 360, 216 (2005), astro-ph/0409162.

(de Lapparent, Geller \& Huchra, 1986) V. de Lapparent, M. J. Geller, J. P. Huchra, A slice of the universe, Astrophys. J. Lett. 302, L1 (1986).

(Dubinski et al., 1993) J. Dubinski, L. N. da Costa, D. S. Goldwirth, M. Lecar, T. Piran, Void evolution and the large-scale structure, Astrophys. J. 410, 458 (1993).

(Eisenstein \& Hu, 1998) D. J. Eisenstein, W. Hu, Baryonic Features in the Matter Transfer Function, Astrophys. J. 496, 605 (1998), astro-ph/9709112.

(Eisenstein \& Hu, 1999) D. J. Eisenstein, W. Hu, Power Spectra for Cold Dark Matter and Its Variants, Astrophys. J. 511, 5 (1999), astro-ph/9710252.

(Erdoğdu et al., 2004) P. Erdoğdu, O. Lahav, S. Zaroubi, G. Efstathiou, S. Moody, J. A. Peacock, M. Colless, I. K. Baldry, C. M. Baugh, J. Bland-Hawthorn, T. Bridges, R. Cannon, S. Cole, C. Collins, W. Couch, G. Dalton, R. De Propris, S. P. Driver, R. S. Ellis, C. S. Frenk, K. Glazebrook, C. Jackson, I. Lewis, S. Lumsden, S. Maddox, D. Madgwick, P. Norberg, B. A. Peterson, W. Sutherland, K. Taylor, The 2dF Galaxy Redshift Survey: Wiener reconstruction of the cosmic web, Mon. Not. R. Astron. Soc. 352, 939 (2004), astro-ph/0312546.

(Falck, Neyrinck \& Szalay, 2012) B. L. Falck, M. C. Neyrinck, A. S. Szalay, ORIGAMI: Delineating Halos Using Phase-space Folds, Astrophys. J. 754, 126 (2012), arXiv:1201.2353 [astro-ph.CO].

(Furlanetto \& Piran, 2006) S. R. Furlanetto, T. Piran, The evidence of absence: galaxy voids in the excursion set formalism, Mon. Not. R. Astron. Soc. 366, 467 (2006), astro-ph/0509148.

(Goldberg \& Vogeley, 2004) D. M. Goldberg, M. S. Vogeley, Simulating Voids, Astrophys. J. 605, 1 (2004), astro-ph/0307191.

(Gregory \& Thompson, 1978) S. A. Gregory, L. A. Thompson, The Coma/A1367 supercluster and its environs, Astrophys. J. 222, 784 (1978).

(Hamaus et al., 2014a) N. Hamaus, P. M. Sutter, G. Lavaux, B. D. Wandelt, Testing cosmic geometry without dynamic distortions using voids, Journal of Cosmology and Astroparticle Physics 12, 013 (2014a), arXiv:1409.3580.

(Hamaus, Sutter \& Wandelt, 2014) N. Hamaus, P. M. Sutter, B. D. Wandelt, Universal Density Profile for Cosmic Voids, Phys. Rev. Lett. 112, 251302 (2014), arXiv:1403.5499.

(Hamaus et al., 2014b) N. Hamaus, B. D. Wandelt, P. M. Sutter, G. Lavaux, M. S. Warren, Cosmology with Void-Galaxy Correlations, Phys. Rev. Lett. 112, 041304 (2014b), arXiv:1307.2571 [astro-ph.CO].

(Heß, Kitaura \& Gottlöber, 2013) S. Heß, F.-S. Kitaura, S. Gottlöber, Simulating structure formation of the Local Universe, Mon. Not. R. Astron. Soc. 435, 2065 (2013), arXiv:1304.6565 [astro-ph.CO].

(Hoffman \& Ribak, 1991) Y. Hoffman, E. Ribak, Constrained realizations of Gaussian fields - A simple algorithm, Astrophys. J. Lett. 380, L5 (1991).

(Icke, 1984) V. Icke, Voids and filaments, Mon. Not. R. Astron. Soc. 206, 1P (1984).

(Ilić, Langer \& Douspis, 2013) S. Ilić, M. Langer, M. Douspis, Detecting the integrated Sachs-Wolfe effect with stacked voids, Astron. \& Astrophys. 556, A51 (2013), arXiv:1301.5849 [astroph.CO].

(Jasche \& Kitaura, 2010) J. Jasche, F. S. Kitaura, Fast Hamilto- nian sampling for large-scale structure inference, Mon. Not. R. Astron. Soc. 407, 29 (2010), arXiv:0911.2496 [astro-ph.CO].

(Jasche et al., 2010a) J. Jasche, F. S. Kitaura, C. Li, T. A. Enßlin, Bayesian non-linear large-scale structure inference of the Sloan Digital Sky Survey Data Release 7, Mon. Not. R. Astron. Soc. 409, 355 (2010a), arXiv:0911.2498 [astro-ph.CO].

(Jasche et al., 2010b) J. Jasche, F. S. Kitaura, B. D. Wandelt, T. A. Enßlin, Bayesian power-spectrum inference for large-scale structure data, Mon. Not. R. Astron. Soc. 406, 60 (2010b), arXiv:0911.2493 [astro-ph.CO].

(Jasche, Leclercq \& Wandelt, 2015) J. Jasche, F. Leclercq, B. D. Wandelt, Past and present cosmic structure in the SDSS DR7 main sample, Journal of Cosmology and Astroparticle Physics 1, 036 (2015), arXiv:1409.6308.

(Jasche \& Wandelt, 2013a) J. Jasche, B. D. Wandelt, Bayesian physical reconstruction of initial conditions from large-scale structure surveys, Mon. Not. R. Astron. Soc. 432, 894 (2013a), arXiv:1203.3639 [astro-ph.CO].

(Jasche \& Wandelt, 2013b) J. Jasche, B. D. Wandelt, Methods for Bayesian Power Spectrum Inference with Galaxy Surveys, Astrophys. J. 779, 15 (2013b), arXiv:1306.1821 [astro-ph.CO].

(Jennings, Li \& Hu, 2013) E. Jennings, Y. Li, W. Hu, The abundance of voids and the excursion set formalism, Mon. Not. R. Astron. Soc. 434, 2167 (2013), arXiv:1304.6087.

(Kirshner et al., 1981) R. P. Kirshner, A. Oemler, Jr., P. L. Schechter, S. A. Shectman, A million cubic megaparsec void in Bootes, Astrophys. J. Lett. 248, L57 (1981).

(Kitaura, 2013) F.-S. Kitaura, The initial conditions of the Universe from constrained simulations, Mon. Not. R. Astron. Soc. 429, L84 (2013), arXiv:1203.4184 [astro-ph.CO].

(Kitaura \& Enßlin, 2008) F. S. Kitaura, T. A. Enßlin, Bayesian reconstruction of the cosmological large-scale structure: methodology, inverse algorithms and numerical optimization, Mon. Not. R. Astron. Soc. 389, 497 (2008), arXiv:0705.0429.

(Kitaura et al., 2009) F. S. Kitaura, J. Jasche, C. Li, T. A. Enßlin, R. B. Metcalf, B. D. Wandelt, G. Lemson, S. D. M. White, Cosmic cartography of the large-scale structure with Sloan Digital Sky Survey data release 6, Mon. Not. R. Astron. Soc. 400, 183 (2009), arXiv:0906.3978 [astro-ph.CO].

(Kitaura, Jasche \& Metcalf, 2010) F.-S. Kitaura, J. Jasche, R. B. Metcalf, Recovering the non-linear density field from the galaxy distribution with a Poisson-lognormal filter, Mon. Not. R. Astron. Soc. 403, 589 (2010), arXiv:0911.1407 [astro-ph.CO].

(Lahav et al., 1994) O. Lahav, K. B. Fisher, Y. Hoffman, C. A. Scharf, S. Zaroubi, Wiener Reconstruction of All-Sky Galaxy Surveys in Spherical Harmonics, Astrophys. J. Lett. 423, L93 (1994), astro-ph/9311059.

(Lavaux, 2010) G. Lavaux, Precision constrained simulation of the local Universe, Mon. Not. R. Astron. Soc. 406, 1007 (2010), arXiv:0912.3525.

(Lavaux \& Wandelt, 2010) G. Lavaux, B. D. Wandelt, Precision cosmology with voids: definition, methods, dynamics, Mon. Not. R. Astron. Soc. 403, 1392 (2010), arXiv:0906.4101 [astroph.CO].

(Lavaux \& Wandelt, 2012) G. Lavaux, B. D. Wandelt, Precision Cosmography with Stacked Voids, Astrophys. J. 754, 109 (2012), arXiv:1110.0345 [astro-ph.CO].

(Leclercq et al., 2013) F. Leclercq, J. Jasche, H. Gil-Marín, B. Wandelt, One-point remapping of Lagrangian perturbation theory in the mildly non-linear regime of cosmic structure formation, Journal of Cosmology and Astroparticle Physics 11, 048 (2013), arXiv:1305.4642 [astro-ph.CO].

(Lee \& Park, 2006) J. Lee, D. Park, Rotation of Cosmic Voids and Void Spin Statistics, Astrophys. J. 652, 1 (2006), astro$\mathrm{ph} / 0606477$.

(Li, Zhao \& Koyama, 2012) B. Li, G.-B. Zhao, K. Koyama, Haloes and voids in $f(R)$ gravity, Mon. Not. R. Astron. Soc. 421, 3481 (2012), arXiv:1111.2602 [astro-ph.CO].

(Li \& Zhao, 2009) B. Li, H. Zhao, Structure formation by a fifth force: $N$-body versus linear simulations, Phys. Rev. D 80, 
044027 (2009), arXiv:0906.3880 [astro-ph.CO].

(Little \& Weinberg, 1994) B. Little, D. H. Weinberg, Cosmic Voids and Biased Galaxy Formation, Mon. Not. R. Astron. Soc. 267, 605 (1994), astro-ph/9306006.

(Martel \& Wasserman, 1990) H. Martel, I. Wasserman, Simulation of cosmological voids in Lambda greater than 0 Friedmann models, Astrophys. J. 348, 1 (1990).

(Melchior et al., 2014) P. Melchior, P. M. Sutter, E. S. Sheldon, E. Krause, B. D. Wandelt, First measurement of gravitational lensing by cosmic voids in SDSS, Mon. Not. R. Astron. Soc. 440, 2922 (2014), arXiv:1309.2045.

(Moutarde et al., 1991) F. Moutarde, J.-M. Alimi, F. R. Bouchet, R. Pellat, A. Ramani, Precollapse scale invariance in gravitational instability, Astrophys. J. 382, 377 (1991).

(Müller et al., 2000) V. Müller, S. Arbabi-Bidgoli, J. Einasto, D. Tucker, Voids in the Las Campanas Redshift Survey versus cold dark matter models, Mon. Not. R. Astron. Soc. 318, 280 (2000), astro-ph/0005063.

(Nadathur \& Hotchkiss, 2014) S. Nadathur, S. Hotchkiss, A robust public catalogue of voids and superclusters in the SDSS Data Release 7 galaxy surveys, Mon. Not. R. Astron. Soc. 440, 1248 (2014), arXiv:1310.2791.

(Nadathur et al., 2014) S. Nadathur, S. Hotchkiss, J. M. Diego, I. T. Iliev, S. Gottlöber, W. A. Watson, G. Yepes, Self-similarity and universality of void density profiles in simulation and SDSS data, ArXiv e-prints (2014), arXiv:1407.1295.

(Neyrinck, 2008) M. C. Neyrinck, ZOBOV: a parameter-free voidfinding algorithm, Mon. Not. R. Astron. Soc. 386, 2101 (2008), arXiv:0712.3049.

(Neyrinck, 2012) M. C. Neyrinck, Origami constraints on the initial-conditions arrangement of dark-matter caustics and streams, Mon. Not. R. Astron. Soc. 427, 494 (2012), arXiv:1202.3364 [astro-ph.CO].

(Neyrinck, 2013) M. C. Neyrinck, Quantifying distortions of the Lagrangian dark-matter mesh in cosmology, Mon. Not. R. Astron. Soc. 428, 141 (2013), arXiv:1204.1326 [astro-ph.CO].

(Neyrinck \& Yang, 2013) M. C. Neyrinck, L. F. Yang, Ringing the initial Universe: the response of overdensity and transformeddensity power spectra to initial spikes, Mon. Not. R. Astron. Soc. 433, 1628 (2013), arXiv:1305.1629 [astro-ph.CO].

(Padilla, Ceccarelli \& Lambas, 2005) N. D. Padilla, L. Ceccarelli, D. G. Lambas, Spatial and dynamical properties of voids in a $\Lambda$ cold dark matter universe, Mon. Not. R. Astron. Soc. 363, 977 (2005), astro-ph/0508297.

(Pan et al., 2012) D. C. Pan, M. S. Vogeley, F. Hoyle, Y.-Y. Choi, C. Park, Cosmic voids in Sloan Digital Sky Survey Data Release 7, Mon. Not. R. Astron. Soc. 421, 926 (2012), arXiv:1103.4156 [astro-ph.CO].

(Park \& Lee, 2007) D. Park, J. Lee, Void Ellipticity Distribution as a Probe of Cosmology, Phys. Rev. Lett. 98, 081301 (2007), astro-ph/0610520.

(Paz et al., 2013) D. Paz, M. Lares, L. Ceccarelli, N. Padilla, D. G. Lambas, Clues on void evolution-II. Measuring density and velocity profiles on SDSS galaxy redshift space distortions, Mon. Not. R. Astron. Soc. 436, 3480 (2013), arXiv:1306.5799 [astroph.CO].

(Pisani et al., 2014) A. Pisani, G. Lavaux, P. M. Sutter, B. D. Wandelt, Real-space density profile reconstruction of stacked voids, Mon. Not. R. Astron. Soc. 443, 3238 (2014), arXiv:1306.3052 [astro-ph.CO].

(Pisani et al., 2015) A. Pisani, P. M. Sutter, N. Hamaus, E. Alizadeh, R. Biswas, B. D. Wandelt, C. M. Hirata, Counting voids to probe dark energy, ArXiv e-prints (2015), arXiv:1503.07690.

(Planck Collaboration, 2014) Planck Collaboration, Planck 2013 results. XIX. The integrated Sachs-Wolfe effect, Astron. \& Astrophys. 571, A19 (2014), arXiv:1303.5079 [astro-ph.CO].

(Platen, van de Weygaert \& Jones, 2007) E. Platen, R. van de Weygaert, B. J. T. Jones, A cosmic watershed: the $W V F$ void detection technique, Mon. Not. R. Astron. Soc. 380, 551 (2007), arXiv:0706.2788.
(Platen, van de Weygaert \& Jones, 2008) E. Platen, R. van de Weygaert, B. J. T. Jones, Alignment of voids in the cosmic web, Mon. Not. R. Astron. Soc. 387, 128 (2008), arXiv:0711.2480.

(Ricciardelli, Quilis \& Varela, 2014) E. Ricciardelli, V. Quilis, J. Varela, On the universality of void density profiles, Mon. Not. R. Astron. Soc. 440, 601 (2014), arXiv:1402.2976 [astro-ph.CO].

(Ryden, 1995) B. S. Ryden, Measuring $Q 0$ from the Distortion of Voids in Redshift Space, Astrophys. J. 452, 25 (1995), astro$\mathrm{ph} / 9506028$.

(Sahni, Sathyaprakah \& Shandarin, 1994) V. Sahni, B. S. Sathyaprakah, S. F. Shandarin, The evolution of voids in the adhesion approximation, Astrophys. J. 431, 20 (1994), astro$\mathrm{ph} / 9403044$.

(Scoccimarro, 2000) R. Scoccimarro, The Bispectrum: From Theory to Observations, Astrophys. J. 544, 597 (2000), astro$\mathrm{ph} / 0004086$.

(Scoccimarro \& Sheth, 2002) R. Scoccimarro, R. K. Sheth, PTHALOS: a fast method for generating mock galaxy distributions, Mon. Not. R. Astron. Soc. 329, 629 (2002), astro-ph/0106120.

(Shandarin, Habib \& Heitmann, 2012) S. Shandarin, S. Habib, K. Heitmann, Cosmic web, multistream flows, and tessellations, Phys. Rev. D 85, 083005 (2012), arXiv:1111.2366 [astro-ph.CO].

(Sheth \& van de Weygaert, 2004) R. K. Sheth, R. van de Weygaert, A hierarchy of voids: much ado about nothing, Mon. Not. R. Astron. Soc. 350, 517 (2004), astro-ph/0311260.

(Shoji \& Lee, 2012) M. Shoji, J. Lee, Voids in Redshift Space, ArXiv e-prints (2012), arXiv:1203.0869 [astro-ph.CO].

(Spolyar, Sahlén \& Silk, 2013) D. Spolyar, M. Sahlén, J. Silk, Topology and Dark Energy: Testing Gravity in Voids, Phys. Rev. Lett. 111, 241103 (2013), arXiv:1304.5239 [astro-ph.CO].

(Springel, 2005) V. Springel, The cosmological simulation code GADGET-2, Mon. Not. R. Astron. Soc. 364, 1105 (2005), astro$\mathrm{ph} / 0505010$.

(Springel, Yoshida \& White, 2001) V. Springel, N. Yoshida, S. D. M. White, GADGET: a code for collisionless and gasdynamical cosmological simulations, New Astronomy 6, 79 (2001), astro-ph/0003162.

(Sutter et al., 2015a) P. M. Sutter, E. Carlesi, B. D. Wandelt, A. Knebe, On the observability of coupled dark energy with cosmic voids, Mon. Not. R. Astron. Soc. 446, L1 (2015a), arXiv:1406.0511.

(Sutter et al., 2015b) P. M. Sutter, G. Lavaux, N. Hamaus, A. Pisani, B. D. Wandelt, M. Warren, F. Villaescusa-Navarro, P. Zivick, Q. Mao, B. B. Thompson, VIDE: The Void IDentification and Examination toolkit, Astronomy and Computing $\mathbf{9}$, 1 (2015b), arXiv:1406.1191.

(Sutter et al., 2014a) P. M. Sutter, G. Lavaux, N. Hamaus, B. D. Wandelt, D. H. Weinberg, M. S. Warren, Sparse sampling, galaxy bias, and voids, Mon. Not. R. Astron. Soc. 442, 462 (2014a), arXiv:1309.5087.

(Sutter et al., 2012a) P. M. Sutter, G. Lavaux, B. D. Wandelt, D. H. Weinberg, A First Application of the Alcock-Paczynski Test to Stacked Cosmic Voids, Astrophys. J. 761, 187 (2012a), arXiv:1208.1058 [astro-ph.CO].

(Sutter et al., 2012b) P. M. Sutter, G. Lavaux, B. D. Wandelt, D. H. Weinberg, A Public Void Catalog from the SDSS DR7 Galaxy Redshift Surveys Based on the Watershed Transform, Astrophys. J. 761, 44 (2012b), arXiv:1207.2524 [astro-ph.CO].

(Sutter et al., 2014b) P. M. Sutter, G. Lavaux, B. D. Wandelt, D. H. Weinberg, M. S. Warren, The dark matter of galaxy voids, Mon. Not. R. Astron. Soc. 438, 3177 (2014b), arXiv:1311.3301 [astro-ph.CO].

(Sutter et al., 2014c) P. M. Sutter, G. Lavaux, B. D. Wandelt, D. H. Weinberg, M. S. Warren, A. Pisani, Voids in the SDSS DR9: observations, simulations, and the impact of the survey mask, Mon. Not. R. Astron. Soc. 442, 3127 (2014c), arXiv:1310.7155.

(Sutter et al., 2014d) P. M. Sutter, A. Pisani, B. D. Wandelt, D. H. Weinberg, A measurement of the Alcock-Paczyński effect using cosmic voids in the SDSS, Mon. Not. R. Astron. Soc. 443, 2983 (2014d), arXiv:1404.5618. 
(Tinker \& Conroy, 2009) J. L. Tinker, C. Conroy, The Void Phenomenon Explained, Astrophys. J. 691, 633 (2009), arXiv:0804.2475.

(van de Weygaert \& Bertschinger, 1996) R. van de Weygaert, E. Bertschinger, Peak and gravity constraints in Gaussian primordial density fields: An application of the Hoffman-Ribak method, Mon. Not. R. Astron. Soc. 281, 84 (1996), astro$\mathrm{ph} / 9507024$.

(van de Weygaert \& Platen, 2011) R. van de Weygaert, E. Platen, Cosmic Voids: Structure, Dynamics and Galaxies, International Journal of Modern Physics Conference Series 1, 41 (2011), arXiv:0912.2997 [astro-ph.CO].

(van de Weygaert \& van Kampen, 1993) R. van de Weygaert, E. van Kampen, Voids in Gravitational Instability Scenarios -
Part One - Global Density and Velocity Fields in an Einstein De-Sitter Universe, Mon. Not. R. Astron. Soc. 263, 481 (1993).

(Viel, Colberg \& Kim, 2008) M. Viel, J. M. Colberg, T.-S. Kim, On the importance of high-redshift intergalactic voids, Mon. Not. R. Astron. Soc. 386, 1285 (2008), arXiv:0801.1105.

(Wang et al., 2013) H. Wang, H. J. Mo, X. Yang, F. C. van den Bosch, Reconstructing the Initial Density Field of the Local Universe: Methods and Tests with Mock Catalogs, Astrophys. J. 772, 63 (2013), arXiv:1301.1348 [astro-ph.CO].

(Zaroubi, 2002) S. Zaroubi, Unbiased reconstruction of the largescale structure, Mon. Not. R. Astron. Soc. 331, 901 (2002), astro-ph/0010561. 